\title{
Improving Classification with Class-Independent Quality Measures: Q-stack in Face Verification
}

\author{
Krzysztof Kryszczuk and Andrzej Drygajlo \\ Swiss Federal Institute of Technology Lausanne (EPFL), Signal Processing Institute \\ http://scgwww.epfl.ch/
}

\begin{abstract}
Existing approaches to classification with signal quality measures make a clear distinction between the single- and multiple classifier scenarios. This paper presents an uniform approach to dichotomization based on the concept of stacking, Q-stack, which makes use of classindependent signal quality measures and baseline classifier scores in order to improve classification in uni- and multimodal systems alike. In this paper we demonstrate the application of Q-stack on the task of biometric identity verification using face images and associated quality measures. We show that the use of the proposed technique allows for reducing the error rates below those of baseline classifiers in single- and multi-classifier scenarios. We discuss how Q-stack can serve as a generalized framework in any single, multiple, and multimodal classifier ensemble.
\end{abstract}

Keywords: statistical pattern classification, quality measures, confidence measures, classifier ensembles, stacking.

\section{Introduction}

Biometric identity verification systems frequently face the challenges of noncontrolled data acquisition conditions. In such conditions biometric signals may suffer from quality degradation due to extraneous, identity-independent factors. It has been demonstrated in numerous reports that a degradation of biometric signal quality is a frequent cause of significant deterioration of classification performance 123], also in multimodal systems, which systematically outperform their unimodal counterparts 4115.

Seeking to improve the robustness of classifiers to degraded data quality, researchers started to incorporate quality measures into the classification process, at the same time making a clear distinction between using quality information in single classifier [2673], as opposed to multi-classifier and multimodal biometric systems [15/8,9]. The application of quality measures in a multimodal system received far more attention, with a dominant intuitive notion that a classifier that has a higher quality data at its disposal ought to be more credible than a classifier that operates on noisy signals [1. In [5] Toh et al. mention that introduction of quality measures provide additional degrees of freedom which helps maximize class separation but neither they explicitly state the actual reason for this improved separation nor do they suggest if and how should this effect 
apply to single classifier systems. However, the impact of signal quality measures on classification performance of a single classifier has also been noticed. In 3. Gales and Young propose to use parallel model architecture to account for varying speech quality in speaker verification. In [26] adaptive threshold selection helps reduce errors. In turn, while quality-dependent model or threshold selection/adaptation is shown to work for single classifiers, the method is not generalized to multiple classifier systems.

We argue that in fact the very same mechanisms governs the use of quality measures in single- and multi-classifier systems alike, and propose a quantitative rather than intuitive perspective on the role of quality measures in classification. In [10] we have proposed $Q$-stack, a novel theoretical framework of improving classification with class-independent quality measures. $Q-$ stack is based on the concept of classifier stacking 11. In the scheme of $Q-$ stack a classifier ensemble is used in which the first classifier layer is made of the baseline unimodal classifiers, and the second, stacked classifier operates on features composed of the normalized similarity scores and the relevant quality measures. The concept of concatenating scores and quality measures into one feature vector was previously used in [9], but only in the context of multimodal fusion using a likelihood ratio-based classifier. In 10 we have demonstrated using synthetic datasets that $Q-$ stack allows for improved class separation using class-independent quality measures for both uni- and multi-modal classification, provided that a statistical dependence exists between the features of the stacked classifier. The nature of the stacked classifier is chosen depending on the actual structure of the classified data. The importance of the dependence between classification scores and quality measures was also stressed in [7, but its implications are not extended beyond error prediction for a single classifier.

In this paper we present $Q$-stack as a general framework of classification with quality measures that is applicable to uni-, multiple-classifier and multimodal biometric verification. We demonstrate the principles and performance of $Q-$ stack on a real biometric dataset, the face part of the BioSec database [12, using two different face image matchers, a quality measure that is correlated with the classification scores of one of the matchers, and three different stacked classifiers. We give evidential support to following hypotheses:

1. A score-dependent quality measure provides an additional dimension in which a stacked classifier can separate the classes better than the baseline classifier that uses only the similarity scores.

2. Proposed method allows to improve biometric verification in single- and multi-classifier scenarios alike.

3. In a multi-classifier system, the quality measure needs to be dependent on at least one classifier in order to observe the benefits of $Q-$ stack.

The paper is structured as follows. Section 2 describes the principles of the proposed method of $Q$ - stack. In Section 3 we treat on the application of $Q$-stack to uni- and multi-classifier face matching, and we give the experimental results with their discussion. Section 4 concludes the paper. 


\section{$2 Q-$ Stack - Using Quality Measures to Improve Classification}

Consider two class data-generating processes $A$ and $B$, which generate features subjected to $k$ arbitrary base classifiers $C_{1,2, \ldots, k}$, each returning a scalar similarity score $x_{1,2, \ldots, k}$. Concatenate these scores to $\mathbf{x}=\left[x_{1}, x_{2}, \ldots, x_{k}\right]$, where vector $\mathbf{x}$ is an instance of a multivariate random variable $X$. The distribution of $X$ is affected by a noise-generating process $N$ that interacts according to some function $\gamma$ with the class-generating processes $A$ and $B$, causing signal degradation. The nature of $\gamma$ needs not be given explicitly [10]. Instead, the interaction between $A, B$ and $N$ manifests itself in the impact of noise instances $n$ on the corresponding observed score instances $\mathbf{x}$. Consequently, a causal dependence between $N$ and $X$ can be observed. In practice it may not be feasible to measure $n$ directly. Instead, a set of $j$ scalar quality measures $\mathbf{q m}=q m_{1}, q m_{2}, \ldots, q m_{j}$ can be collected, where $\mathbf{q m}$ denotes an instance of a random variable $Q M$. By definition, $Q M$ is dependent on $N$, and therefore it also inherits a dependence on $X$. At the same time, quality measures do not carry class-selective information, $p(\mathbf{q m} \mid A)=p(\mathbf{q m} \mid B)$.

Let us now concatenate the training scores $\mathbf{x}$ and the relevant quality measures $\mathbf{q m}$ into evidence vectors $\mathbf{e}=[\mathbf{x}, \mathbf{q m}]$, and analyze the separation between classes $A$ and $B$ in the $(k+j)$-dimensional evidence space defined by all components of the evidence vectors. Under the assumption of equal priors, $P(A)=P(B)$, class separation can be expressed in terms of divergence between class-conditional joint distributions of $p(\mathbf{e} \mid A)$ and $p(\mathbf{e} \mid B)$. In [13] Koval et al. have shown that divergence between joint class-conditional distributions is greater for dependent, than for independent classification features. Consequently, since $p(Q M \mid A)=$ $p(Q M \mid B)$ the existence of statistical dependencies between $X$ and $Q M$ grants that

$$
\int_{-\infty}^{\infty}|p(\mathbf{x} \mid B)-p(\mathbf{x} \mid A)| d \mathbf{x}<\int_{-\infty}^{\infty}|p(\mathbf{e} \mid B)-p(\mathbf{e} \mid A)| d \mathbf{e}
$$

A detailed proof of (10) is beyond the frames of this paper. An intuitive understanding of this result is shown in Figure 1. Here, the evidence consists of one class-selective score and one quality measure, $\mathbf{e}=[x, q m]$. In both subplots the marginal class-conditional distributions of evidence remain unchanged. The variables of $X$ and $Q M$ are independent in the left, and dependent in the right subplot. Note that in the independent case the class separation is defined entirely by $p(\mathbf{x} \mid A)$ and $p(\mathbf{x} \mid B)$. In the presence of a dependence between $X$ and $Q M$ classes $A$ and $B$ are clearly better separated. For more details the reader is referred to 10 .

As a consequence of (1), classification in the evidence space is guaranteed to be more accurate than using base scores $\mathbf{x}$ alone, as long as there is a dependence between $X$ and $Q M$. For classification in the evidence space the scores of the base classifiers $\mathbf{x}$ become part of the feature vector for a new stacked classifier 11, hence the coined name $Q-$ stack. The stacked classifier can be chosen arbitrarily, depending on the actual joint distributions of evidence e. 

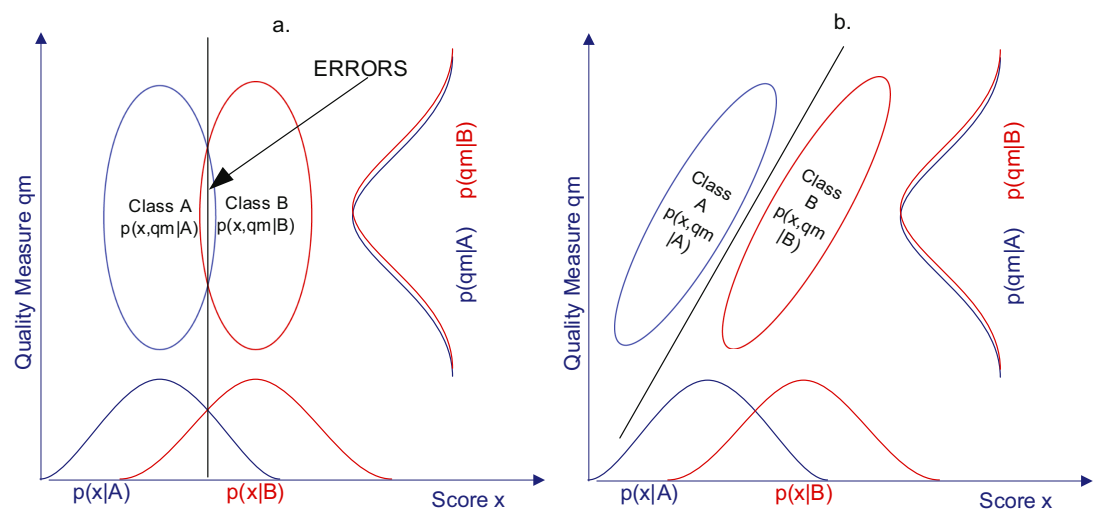

Fig. 1. Graphical representation of the impact of dependence between scores $x$ and quality measures $q m$ on class separation in the evidence space $e=[x, q m]$ : a. independent $x$ and $q m$, b. dependent $x$ and $q m$.

Figure 2 shows a block diagram of $Q-$ stack applied in a single-classifier scenario. Features extracted from a single signal are classified by a single base classifier. At the same time, quality measures (one or more) are collected. Classification scores and quality measures are combined into evidence vectors and classified by a stacked classifier operating in the evidence space. In Figure 3 the very same structure is applied to multimodal classification. Two signals are classified in parallel, resulting in score vector $\mathbf{x}$, which is further combined together with the respective quality measures into an evidence vector $\mathbf{e}=[\mathbf{x}, \mathbf{q m}]$. The evidence vector e becomes a feature vector for the stacked classifier. Note that if no quality measures are present, the architecture shown in Figure 3 simply performs a multimodal score-level fusion.

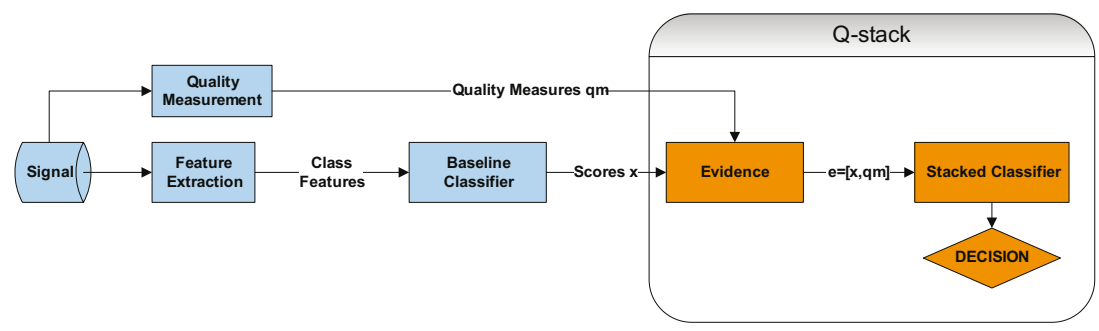

Fig. 2. Q-stack in single base classifier scenario

\section{Q-Stack for Face Verification}

The experiments presented here give an embodiment of the proposed method of $Q$ - stack. We show that the improvements in the system performance do indeed hinge on the statistical dependencies of the signal quality measures on 


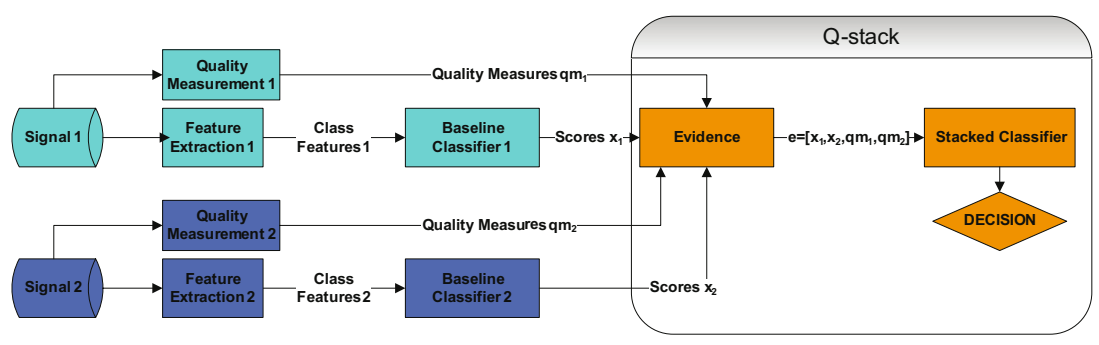

Fig. 3. Q-stack in multimodal/multiclassifier scenario

the corresponding classifier scores, using an example of face matching. In our experiments we used face images of 200 subjects (training set: 50 subjects, testing set: 150 subjects) from the BioSec database, baseline corpus. For the details regarding the BioSec database the reader is referred to [12. The experiments presented in this paper involve one-to-one sample matching. All face images have been registered manually in order to avoid the impact of face localization algorithms on the matching performance. All images have been photometrically normalized [14.

In our experiments we used the following two face matchers: $1 . D C T$ - local DCT mod2 features and a Bayes classifier based on the feature distributions approximated by Gaussian Mixture Models (GMM) 15] (scores produced by the $D C T$ matcher denoted as $\left.x_{1}\right)$, 2. PCA - Mahalanobis distance between global $P C A$ feature vectors [16. The $P C A$ projection space was found using all images from the training dataset. The scores produced by the $P C A$ matcher are denoted as $x_{2}$. The two face matchers were chosen since they both operate on very different features. The local DCTmod2 features encode mostly high spacial frequencies, while the projection of the face images on the $P C A$ subspace emphasizes lower spacial frequencies.

In order to test the hypothesis that a quality measure needs to sport a statistical dependence on the classification scores, for the experiments reported here a quality measure $Q M$ was chosen that correlates well with the scores of one of the used matchers, but not both. Namely, we used a normalized 2-dimensional cross correlation with an average face template [17, which due to its nature was expected to be dependent on the scores $x_{2}$ of the $P C A$ classifier. If $q m_{\alpha}$ and $q m_{\beta}$ are the quality measures computed for each of the matched face images then the resulting combined quality measure used as evidence was computed as $q m=\sqrt{q m_{\alpha} q m_{\beta}}$, following [1].

In order to maintain a consistent notation with Section 2 and with [10] denote here the class of genuine client match scores as Class $A$, and the class of the imposter match scores as Class $B$. The class-conditional distributions of classifier scores $x_{1}$ (DTC matcher), $x_{2}$ (PCA matcher), and quality measures $Q M$ are shown in Figure 4 The distributions of the quality measures in Figure $4 c$ show that indeed the used quality measures are class-independent and cannot help separate classes on their own. 

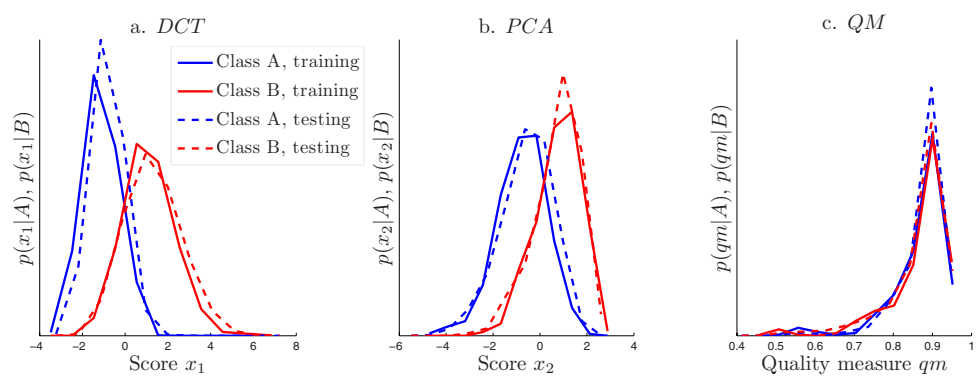

Fig. 4. Class-conditional distributions of scores $x_{1}, x_{2}$ and quality measures $q m$

Table 1. Correlation coefficients between evidence components $x_{1}, x_{2}, q m$

\begin{tabular}{|l|ccc||c|ccc|}
\hline \multicolumn{3}{|c||}{ Training } & \multicolumn{4}{c|}{ Testing } \\
\hline & $x_{1}$ & $x_{2}$ & $q m$ & & $x_{1}$ & $x_{2}$ & $q m$ \\
\hline$x_{1}$ & 1.00 & 0.57 & 0.02 & $x_{1}$ & 1.00 & 0.36 & -0.11 \\
\hline$x_{2}$ & 0.57 & 1.00 & 0.46 & $x_{2}$ & 0.36 & 1.00 & 0.50 \\
\hline$q m$ & 0.02 & 0.46 & 1.00 & $q m$ & -0.11 & 0.50 & 1.00 \\
\hline
\end{tabular}

As we have discussed before, a quality measure needs to be dependent on the scores in order to improve the class separation. Since correlation between random variables entails dependence, we computed the pair-wise Pearson's correlation coefficients between $x_{1}, x_{2}$ and $q m$ for both the training and testing data sets see Table1 A strong correlation is evident between the scores $x_{1}$ and $x_{2}$, which is a consequence of the fact that both classifiers operate on the same input signals. However, the quality measure $Q M$ is strongly correlated only with the scores $x_{2}$ originating from the $P C A$ classifier.

The experiments were conducted as follows. First, the baseline classifiers $D C T$ and $P C A$ were trained using the dedicated training data set of the BioSec database, disjoint from the testing set, originating from a separate set of users, and quality measures $q m$ were collected. The proposed method of $Q-$ stack was applied to evidence vectors composed of $\mathbf{e}=\left[x_{1}, q m\right], \mathbf{e}=\left[x_{2}, q m\right], \mathbf{e}=\left[x_{1}, x_{2}\right]$ and $\mathbf{e}=\left[x_{1}, x_{2}, q m\right]$. The combinations of $\mathbf{e}=\left[x_{1}, q m\right]$ and $\mathbf{e}=\left[x_{2}, q m\right]$ are examples of $Q$-stack applied to a single classifier. The evidence vector $e=\left[x_{1}, x_{2}\right]$ is $Q$-stack with only score-level evidence from two classifiers, which is equivalent to multi-classifier fusion [4. Finally, the evidence vector $\mathbf{e}=\left[x_{1}, x_{2}, q m\right]$ represents $Q$-stack applied to multi-classifier fusion with quality measures. Scores $x_{1}$, $x_{2}$ and quality measures $q m$ were normalized to zero mean and unit variance using normalization parameters estimated on the training dataset. Three different stacked classifiers that separated $e \mid A$ from $e \mid B$ were used: Support Vector Machines classifier with a Radial Base Function kernel $(S V M)$, a Bayes classifier using Gaussian approximations of the class-conditional distributions (Bayes), and a Linear Discriminant-based classifier $(L D A)$. 

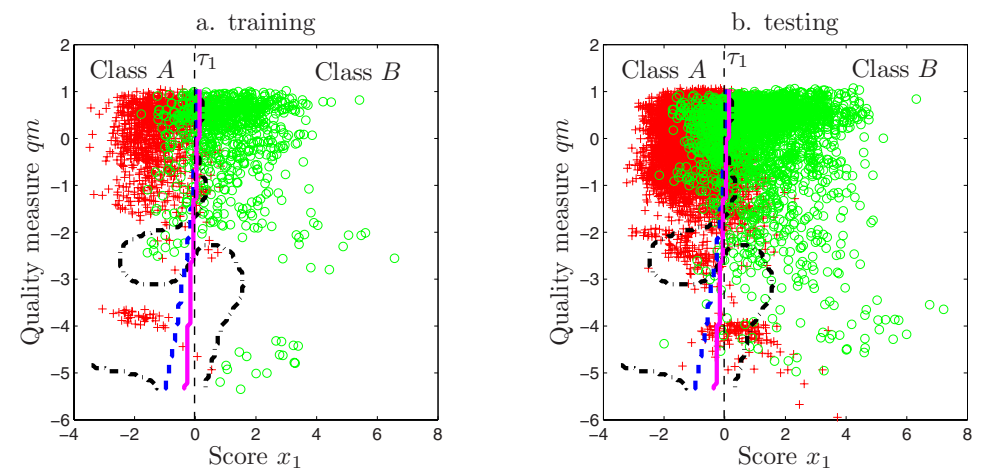

Fig. 5. Class separation in the evidence space $\mathbf{e}=\left[x_{1}, q m\right]$ for the $D C T$ classifier. Bold lines show the $Q-$ stack decision boundaries for following stacked classifiers: SVM (dash-dot), Bayes (dashed) and LDA (solid). Thin dashed line marked $\tau_{1}$ shows the corresponding decision boundary of the baseline $D C T$ classifier.

Figure 5 shows the application of $Q-$ stack to the $D C T$ classifier with evidence vector $\mathbf{e}=\left[x_{1}, q m\right]$ and Figure 6 shows the application of $Q-$ stack to the $P C A$ classifier, evidence vector $\mathbf{e}=\left[x_{1}, q m\right]$ for both the training $(a)$ and testing (b) datasets. The solid lines in both figures show the class decision boundaries for the corresponding stacked classifiers, optimized on the testing dataset. The thin dashed lines marked $\tau_{1}$ and $\tau_{2}$ represent baseline classification boundaries for $x_{1}$ and $x_{2}$, respectively. In Figures 5 and 6 the areas confined by the bold $(Q-$ stack $)$ and the thin lines show the loci of observations that can be classified more accurately by $Q-$ stack than by the baseline classifiers $\left(\tau_{1}\right.$ and $\left.\tau_{2}\right)$. The gains in accuracy due to the classification in the space of evidence $\mathbf{e}$ rather than in the score space $x_{1}$ or $x_{2}$ are summarized in Table 2. Classification results are reported in terms of total classification accuracy $A C$, Half-Total Error Rate $H T E R$ [4] and error rates for individual classes $E R_{A}$ and $E R_{B}$, obtained on the testing dataset.

A weak correlation between the quality measure and the scores $x_{1}$ of the $D C T$ classifier (Table 1) result in marginal improvements in classification accuracy. Indeed, as it is shown in Figure 5 the $Q$ - stack decision boundary does not deviate much from the baseline classification boundary given by $x_{1}=\tau_{1}$. The exception here is the stacked $S V M$ classifier, whose shape of the decision boundary suggests overfitting. Strong correlation between the the quality measure $Q M$ and the scores $x_{2}$ of the $P C A$ classifier is reflected in a $Q-$ stack decision boundaries that deviate consistently and significantly from that of the baseline classifier given by $x_{2}=\tau_{2}$ (Figure 6). Large areas confined by the $Q-$ stack and $\tau_{2}$ decision bounds suggest a significant improvement of classification accuracy in the $\mathbf{e}=\left[x_{2}, q m\right]$ space compared with the baseline classifier. This improvement is indeed consistent for all stacked classifiers.

In the case of classification in the $\mathbf{e}=\left[x_{1}, x_{2}\right]$ space, $Q-$ stack is equivalent to any trained classifier fusion method: here using SVM, Bayesian and LDA classifiers. The results shown in Table 2 show an improvement in classification 
a. training

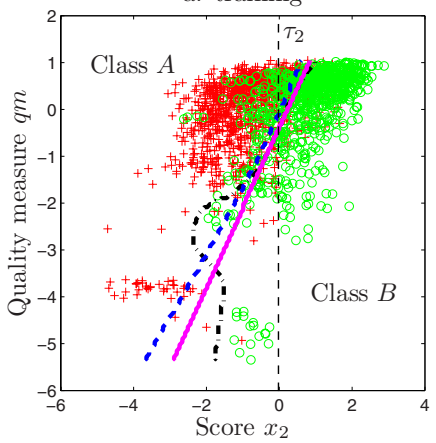

b. testing

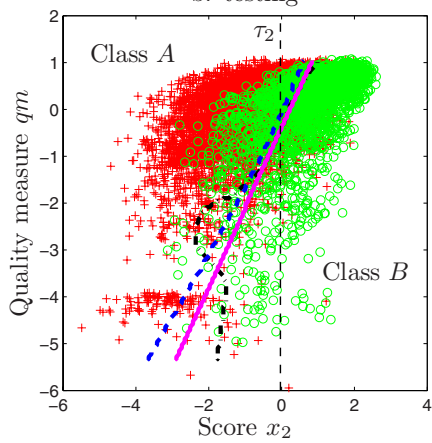

Fig. 6. Class separation in the evidence space $\mathbf{e}=\left[x_{2}, q m\right]$ for the $P C A$ classifier. Bold lines show the $Q$ - stack decision boundaries for following stacked classifiers: SVM (dash-dot), Bayes (dashed) and LDA (solid). Thin dashed line marked $\tau_{2}$ shows the corresponding decision boundary of the baseline $P C A$ classifier.

Table 2. Comparison of classification performance achieved by the baseline classifiers and using the proposed $Q$-stack method. Experimental results reported for the $S V M$, Bayes and $L D A$ stacked classifiers operating in the evidence space.

\begin{tabular}{|c|c|c|c|c|}
\hline & \multicolumn{4}{|c|}{\begin{tabular}{|l|l|l|l||} 
Accuracy $[\%]$ & $H T E R[\%]$ & $E R_{A}[\%]$ & $E R_{B}[\%]$ \\
\end{tabular}} \\
\hline \multicolumn{5}{|c|}{$\overline{\overline{\text { Baseline }}}$} \\
\hline$D C T: \mathbf{e}=\left[x_{1}\right]$ & 83.97 & 14.92 & 13.21 & 16.64 \\
\hline$P C A: \mathbf{e}=\left[x_{2}\right]$ & 70.62 & 26.68 & 15.25 & 13.36 \\
\hline \multicolumn{5}{|c|}{ SVM } \\
\hline$\overline{D C T+Q M: \mathbf{e}=\left[x_{1}, q m\right]}$ & 84.97 & 14.95 & 14.83 & 15.07 \\
\hline$P C A+Q M: \mathbf{e}=\left[x_{2}, q m\right]$ & 79.23 & 20.68 & 20.54 & 20.82 \\
\hline$D C T+P C A: \mathbf{e}=\left[x_{1}, x_{2}\right]$ & 87.41 & 12.73 & 12.96 & 12.51 \\
\hline$D C T+P C A+Q M: \mathbf{e}=\left[x_{1}, x_{2}, q m\right]$ & 87.78 & 12.12 & 11.96 & 12.28 \\
\hline \multicolumn{5}{|c|}{ LDA } \\
\hline$\overline{D C T+Q M: \mathbf{e}=\left[x_{1}, q m\right]}$ & 83.85 & 14.83 & 12.79 & 16.88 \\
\hline$P C A+Q M: \mathbf{e}=\left[x_{2}, q m\right]$ & 77.32 & 21.53 & 19.75 & 23.31 \\
\hline$D C T+P C A: \mathbf{e}=\left[x_{1}, x_{2}\right]$ & 85.91 & 13.06 & 11.46 & 14.66 \\
\hline$D C T+P C A+Q M: \mathbf{e}=\left[x_{1}, x_{2}, q m\right]$ & 88.68 & 11.57 & 11.96 & 11.19 \\
\hline \multicolumn{5}{|c|}{ Bayes } \\
\hline$D C T+Q M: \mathbf{e}=\left[x_{1}, q m\right]$ & 84.85 & 14.65 & 13.88 & 15.43 \\
\hline$P C A+Q M: \mathbf{e}=\left[x_{2}, q m\right]$ & 76.41 & 21.56 & 18.42 & 24.7 \\
\hline$D C T+P C A: \mathbf{e}=\left[x_{1}, x_{2}\right]$ & 86.23 & 13.04 & 11.92 & 14.17 \\
\hline$D C T+P C A+Q M: \mathbf{e}=\left[x_{1}, x_{2}, q m\right]$ & 87.03 & 12.3 & 11.25 & 13.34 \\
\hline
\end{tabular}

accuracy over both baseline systems, which is an expected result. The classification results for $\mathbf{e}=\left[x_{1}, x_{2}\right]$ serve as multi-classifier baseline for the comparison with the results obtained by applying $Q-$ stack to fusion with $\mathbf{e}=\left[x_{1}, x_{2}, q m\right]$. Despite the fact that $Q M$ correlates well only with $x_{2}$ but not with $x_{1}$, adding the quality measure to the evidence vector $\left(\mathbf{e}=\left[x_{1}, x_{2}, q m\right]\right)$ resulted in fur- 
ther consistent improvements in classification performance. $Q-$ stack applied to multi-classifier fusion with quality measures proved to deliver the lowest error rates of all compared systems.

\section{Conclusions}

In this paper we have presented $Q$ - stack, a uniform method of incorporating class-independent quality measures in unimodal classification and multi-classifier systems. The method is based on the improved class separation due to the dependence between the classifier scores and the quality measures. We have demonstrated the method to be effective in improving both single- and multi-classifier face verification. We have also shown that the benefits that can be expected of the application of $Q$ - stack hinge on the statistical dependencies between the quality measures and the baseline classifier similarity scores, and on a proper selection of the stacked classifier according to the class-conditional evidence distributions. The method generalizes well to other modalities - we have obtained promising results also for fingerprint and speech modalities. Multiple quality measures can be incorporated by simply adding them into the evidence vector. The results suggest that particular attention must be paid to the development of classifier-quality measure ensembles, rather than classifier-independent quality measures alone.

\section{Acknowledgments}

This work was partially funded by the Swiss National Science Foundation $(S N S F)$ and National Centre of Competence in Research (NCCR)IM2.MPR. We wish to thank Prof. Javier Garcia-Ortega (UAM) for making the face part of the BioSec database available for our experiments.

\section{References}

1. Fierrez-Aguilar, J.: Adapted Fusion Schemes for Multimodal Biometric Authentication. PhD thesis, Universidad Politecnica de Madrid (2006)

2. Kryszczuk, K., Drygajlo, A.: Gradient-based image segmentation for face recognition robust to directional illumination. In: Visual communications and image processing 2005, Beijing, China, July 12-15, 2005 (2005)

3. Gales, M.J.F., Young, S.J.: Robust continuous speech recognition using parallel model compensation. IEEE Transactions on Acoustics, Speech and Signal Processing 4(5) (1996)

4. Bengio, S., Marcel, C., Marcel, S., Mariethoz, J.: Confidence measures for multimodal identity verification. Information Fusion 3(4), 267-276 (2002)

5. Toh, K.A., Yau, W.Y., Lim, E., Chen, L., Ng, C.H.: Fusion of auxiliary information for multi-modal biometrics authentication. In: Proceedings of International Conference on Biometrics, Hong Kong. LNCS, pp. 678-685. Springer, Heidelberg (2004) 
6. Wein, L., Baveja, M.: Using fingerprint image quality to improve the identification performance of the U.S. VISIT program. In: Proceedings of the National Academy of Sciences (2005)

7. Grother, P., Tabassi, E.: Performance of biometric quality measures. IEEE Transactions on Pattern Analysis and Machine Intelligence 29(4), 531-543 (2007)

8. Poh, N., Heusch, G., Kittler, J.: On combination of face authentication experts by a mixture of quality dependent fusion classifiers. In: Proceedings of th 7 th International Workshop on Multiple Classifier Systems, Prague, Czech Republic (2007)

9. Nandakumar, K., Chen, Y., Dass, S.C., Jain, A.K.: Quality-based score level fusion in multibiometric systems. In: Proceedings of International Conference on Pattern Recognition, Hong Kong, China, vol. 4, pp. 473-476 (2006)

10. Kryszczuk, K., Drygajlo, A.: Q-stack: uni- and multimodal classifier stacking with quality measures. In: Proceedings of the International Workshop on Multiple Classifier Systems, Prague, Czech Republic (2007)

11. Wolpert, D.: Stacked generalization. Neural Networks 5, 241-259 (1992)

12. Fierrez, J., Ortega-Garcia, J., Torre-Toledano, D., Gonzalez-Rodriguez, J.: Biosec baseline corpus: A multimodal biometric database. Pattern Recognition 40(4), 1389-1392 (2007)

13. Koval, O., Voloshynovskiy, S., Pun, T.: Error exponent analysis of person identification based on fusion of dependent/independent modalities. In: Proceedings of SPIE Photonics West, Electronic Imaging 2006, Multimedia Content Analysis, Management, and Retrieval 2006 (EI122) (2006)

14. Gross, R., Brajovic, V.: An image preprocessing algorithm for illumination invariant face recognition. In: Kittler, J., Nixon, M.S. (eds.) AVBPA 2003. LNCS, vol. 2688, Springer, Heidelberg (2003)

15. Sanderson, C.: Automatic Person Verification Using Speech and Face Information. PhD thesis, Griffith University, Queensland, Australia (2003)

16. Turk, M.A., Pentland, A.P.: Eigenfaces for recognition. Journal of Cognitive Neuroscience 3(1), 71-86 (1991)

17. Kryszczuk, K., Drygajlo, A.: On combining evidence for reliability estimation in face verification. In: Proc. of the EUSIPCO 2006, Florence (September 2006) 